\title{
Design and Stability Analysis of a Fuzzy Adaptive SMC System for Three-Phase UPS Inverter
}

\author{
Khawar Naheem ${ }^{*}$, Young-Sik Choi ${ }^{*}$, Francis Mwasilu*, Han Ho Choi ${ }^{*}$, and Jin-Woo Jung ${ }^{\dagger}$ \\ ${ }^{* \dagger}$ Division of Electronics and Electrical Engineering, Dongguk University, Seoul, Korea
}

\begin{abstract}
This paper proposes a combined fuzzy adaptive sliding-mode voltage controller (FASVC) for a three-phase UPS inverter. The proposed FASVC encapsulates two control terms: a fuzzy adaptive compensation control term, which solves the problem of parameter uncertainties, and a sliding-mode feedback control term, which stabilizes the error dynamics of the system. To extract precise load current information, the proposed method uses a conventional load current observer instead of current sensors. In addition, the stability of the proposed control scheme is fully guaranteed by using the Lyapunov stability theory. It is shown that the proposed FASVC can attain excellent voltage regulation features such as a fast dynamic response, low total harmonic distortion (THD), and a small steady-state error under sudden load disturbances, nonlinear loads, and unbalanced loads in the existence of the parameter uncertainties. Finally, experimental results are obtained from a prototype $1 \mathrm{kVA}$ three-phase UPS inverter system via a TMS320F28335 DSP. A comparison of these results with those obtained from a conventional sliding-mode controller (SMC) confirms the superior transient and steady-state performances of the proposed control technique.
\end{abstract}

Key words: Fuzzy Adaptive Sliding-mode Voltage Controller (FASVC), Three-phase inverter, Total Harmonic Distortion (THD), Uninterruptible Power Supply (UPS)

\section{INTRODUCTION}

Nowadays, the nonlinear nature of electric loads is resulting in a strong demand for high-quality and reliable power sources from both customers and utilities [1]-[5]. To address this issue, uninterruptible power supplies (UPSs) are being extensively employed for critical loads such as communication systems, medical support systems, emergency systems, etc. [6]. For improving power quality through UPS systems, it is important to achieve sinusoidal output voltage waveforms with a fast voltage recovery capability and a very low total harmonic distortion (THD) regardless of the type of load. Typically, an inverter with an $L C$ output filter in a UPS system is a suitable solution to fulfill this requirement. The main criteria for evaluating the regulation performance of the UPS inverter output voltage are a quick dynamic response, low THD, and small steady-state error. Moreover, various load conditions such as abrupt load

Manuscript received Feb. 16, 2014; accepted May 8, 2014

Recommended for publication by Associate Editor Jun-Keun Ji. †Corresponding Author: jinwjung@dongguk.edu

Tel: +82-2-2260-3348, Dongguk University

*Division of Electronics and Electrical Engineering, Dongguk University, Korea changes, nonlinear loads, and unbalanced loads seriously harm the performance of UPS inverters. Thus, an appropriate control strategy is desired to sufficiently meet the performance criteria of UPS systems under any type of electrical load.

Recently, many researchers have presented a number of advanced control techniques for UPS systems [7]-[20]. The authors of [7] described a feedback linearization control scheme for the UPS inverter. This method focuses on achieving a low THD and a fast dynamic response without considering parameter uncertainties. In [8] and [9], a repetitive control is applied to generate a high-quality sinusoidal output voltage. In general, this technique has problems such as a slow transient response and instability to error dynamics. In [10], a model predictive control is suggested for regulating the UPS output voltage. This method utilizes a load current observer and has a small steady-state error. However, it reflects a high THD value in the output voltage under linear and nonlinear loads. In [11], a hybrid PID control scheme with multiple loops is proposed. With the advantages of ensuring a good performance and easy implementation, these techniques require many trials to tune for the proper gains. According to [12], a deadbeat control technique can provide a fast dynamic response and high 
accuracy. However, this scheme is very sensitive to the parameter uncertainties. In [13], an $H_{\infty}$ loop-shaping control scheme is presented. This method has a simple structure and is robust under model uncertainties. Nevertheless, it is applied to a single-phase inverter. In [14], an adaptive fuzzy control method is illustrated for a three-phase UPS system. Although this approach tracks the desired sinusoidal waveform regardless of being subjected to nonlinear loads, the large number of fuzzy rules employed raises the computational burden. In [15], a hybrid fuzzy-repetitive control is applied for the UPS inverter. This scheme reveals a good transient response under load disturbance; whereas it is applied to a single-phase UPS inverter and its THD value is high for nonlinear loads. Next, a sliding-mode control method is employed on UPS inverters [16]-[20]. It is obvious from [16]-[18] that good UPS performance can be obtained by this control scheme. However, in [16], [17], the control scheme is only implemented on a single-phase inverter and in [18] the results for nonlinear loads are not provided. In [19] and [20], the authors achieve a good voltage response, but the stability analysis is not presented.

This paper presents a fuzzy adaptive sliding-mode voltage controller (FASVC) for a three-phase UPS inverter. In this paper, the solution to parameter uncertainties is provided by a fuzzy adaptive compensation control term. In addition, the error dynamics of the system are stabilized by a sliding-mode feedback control term. A conventional load current observer is utilized to precisely estimate the load-current without using current sensors. The stability of the proposed FASVC is completely validated by the Lyapunov theory. Hence, in real applications, the proposed control technique can accomplish exceptional voltage regulation performance (such as a fast dynamic response, low THD, and small steady-state error) not only in the presence of parameter uncertainties, but also under sudden load changes, nonlinear loads, and unbalanced loads. A conventional sliding-mode controller (SMC) is also tested to highlight the outstanding performance of the suggested control approach. Finally, the validity of the proposed FASVC is demonstrated by comparative experimental results carried out on a prototype $1 \mathrm{kVA}$ three-phase UPS inverter system using a TMS320F28335 DSP.

\section{Mathematical Modeling OF Three-Phase UPS INVERTER SYSTEM}

A circuit diagram of the three-phase UPS inverter system with an $L C$ output filter is shown in Fig. 1. It consists of the following components: a DC-link $\left(V_{D C}\right)$, a three-phase IGBT inverter, three filter capacitors $\left(C_{f}\right)$, three filter inductors $\left(L_{f}\right)$, and a three-phase load.

The state equations of Fig. 1 can be represented in the synchronously rotating $d q$ reference frame by the following system model equations [7]:

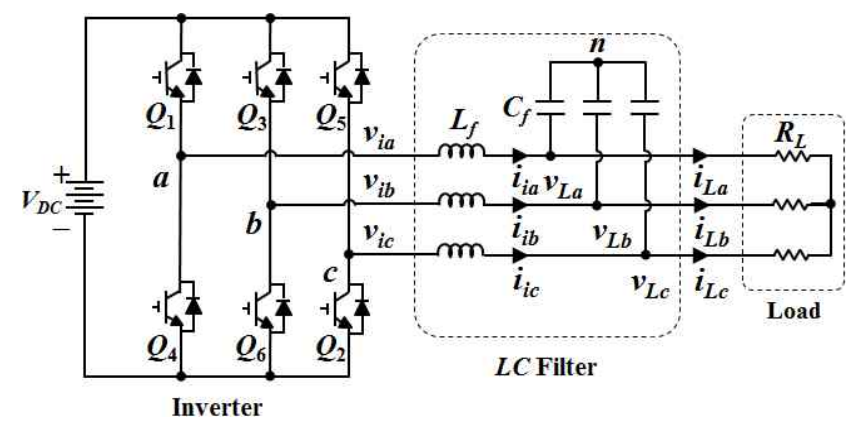

Fig. 1. Circuit diagram of a three-phase UPS inverter system.

$$
\begin{aligned}
& \dot{i}_{i d}=-\frac{1}{L_{f}} v_{L d}+\frac{1}{L_{f}} v_{i d}+\omega i_{i q} \\
& \dot{i}_{i q}=-\frac{1}{L_{f}} v_{L q}+\frac{1}{L_{f}} v_{i q}-\omega i_{i d} \\
& \dot{v}_{L d}=-\frac{1}{C_{f}} i_{L d}+\frac{1}{C_{f}} i_{i d}+\omega v_{L q} \\
& \dot{v}_{L q}=-\frac{1}{C_{f}} i_{L q}+\frac{1}{C_{f}} i_{i q}-\omega v_{L d}
\end{aligned}
$$

where $i_{i d}, i_{i q}, v_{i d}$, and $v_{i q}$ are the $d q$-axis inverter currents and voltages, $i_{L d}, i_{L q}, v_{L d}$, and $v_{L q}$ are the $d q$-axis load currents and voltages, and $\dot{i}_{i d}, \dot{i}_{i q}, \dot{v}_{L d}$, and $\dot{v}_{L q}$ are the time derivatives of the $d q$-axis inverter currents and load voltages, respectively. In addition, $\omega$ denotes the system angular frequency $(\omega=2 \pi f)$, and $f$ is the fundamental frequency of the load voltage.

It should be noted that:

1) $\boldsymbol{i}_{i d q}$ and $\boldsymbol{v}_{L d q}$ are the state variables and measurable;

2) $\boldsymbol{v}_{i d q}$ is the control input;

3) $\boldsymbol{i}_{L d q}$ is the unknown disturbance and changes very slowly during a sampling period [10];

4) The desired $d q$-axis load voltages $v_{L d}{ }^{*}$ and $v_{L q}{ }^{*}$ are constant during a sampling period and then their time derivatives can be set to zero.

\section{FuZZY ADAPTIVE SLIDING-Mode Voltage CONTROLLER DESIGN AND STABILITY ANALYSIS}

This section thoroughly presents the proposed FASVC algorithm and its stability analysis by utilizing the system model (1). First, the errors of the $d q$-axis inverter currents $\left(i_{i d}\right.$, $\left.i_{i q}\right)$ and load voltages $\left(v_{L d}, v_{L q}\right)$ are defined as follows:

$$
\begin{aligned}
e_{i d}=i_{i d}-i_{i d} *, & e_{i q}=i_{i q}-i_{i q} * \\
i_{i d} *=i_{L d}-\omega C_{f} v_{L q}, & i_{i q} *=i_{L q}+\omega C_{f} v_{L d} \\
e_{L d}=v_{L d}-v_{L d} *, & e_{L q}=v_{L q}-v_{L q} *
\end{aligned}
$$

where $e_{i d}, e_{i q}, i_{i d}{ }^{*}$, and $i_{i q}{ }^{*}$ are the errors and reference values of the $d q$-axis inverter currents, respectively. Similarly, $e_{L d}$, $e_{L q}, v_{L d}{ }^{*}$, and $v_{L q}{ }^{*}$ are the errors and reference values of the $d q$-axis load voltages, respectively. Then, the following error dynamics can be derived: 


$$
\begin{gathered}
\dot{e}_{i d}=-\frac{1}{L_{f}}\left(u_{d}-v_{i n d}\right)+\frac{1}{\gamma_{d}} \frac{1}{C_{f}} e_{i d} \\
\dot{e}_{i q}=-\frac{1}{L_{f}}\left(u_{q}-v_{i n q}\right)+\frac{1}{\gamma_{q}} \frac{1}{C_{f}} e_{i q} \\
\dot{e}_{L d}=\frac{1}{C_{f}} e_{i d}, \quad \dot{e}_{L q}=\frac{1}{C_{f}} e_{i q}
\end{gathered}
$$

where $u_{d}, u_{q}, v_{\text {ind }}$, and $v_{i n q}$ are the system uncertainty terms and the control inputs, respectively, and $\gamma_{d}$ and $\gamma_{q}$ denote positive numbers.

The system uncertainty terms $\left(u_{d}, u_{q}\right)$ are given by:

$$
\begin{aligned}
u_{d}= & -\frac{\omega L_{f}}{\gamma_{d}} v_{L q}+\frac{L_{f}}{C_{f} \gamma_{d}} i_{L d}-\frac{L_{f}}{C_{f} \gamma_{d}} i_{i d} \\
& -L_{f} \omega i_{i q}+L_{f} i_{i d} *+v_{L d} \\
u_{q}= & \frac{\omega L_{f}}{\gamma_{q}} v_{L d}+\frac{L_{f}}{C_{f} \gamma_{q}} i_{L q}-\frac{L_{f}}{C_{f} \gamma_{q}} i_{i q} \\
& +L_{f} \omega i_{i d}+L_{f} \dot{i}_{i q} *+v_{L q}
\end{aligned}
$$

It should be noticed from (4) that $u_{d}$ and $u_{q}$ contain the time derivative terms $\left(\dot{i}_{i d} *, \dot{i}_{i q} *\right)$ that cannot be computed in a straightforward manner due to system noises. In addition, it is assumed that $L_{f}$ has some uncertainties because of the nonlinear magnetic properties. In practice, the exact estimation of the uncertainty terms $\left(u_{d}, u_{q}\right)$ is required instead of directly using the time derivatives of $i_{i d}{ }^{*}$ and $i_{i q}{ }^{*}$.

Next, the control inputs $\left(v_{i n d}, v_{i n q}\right)$ can be written as:

$$
\left[\begin{array}{c}
v_{i n d} \\
v_{i n q}
\end{array}\right]=\left[\begin{array}{l}
u_{f a d}+u_{s m d} \\
u_{f a q}+u_{s m q}
\end{array}\right]
$$

where $u_{f a d}, u_{f a q}, u_{s m d}$, and $u_{s m q}$ are the $d q$-axis fuzzy adaptive compensation control terms and the $d q$-axis sliding-mode feedback control terms, respectively.

Proposition 1: Assume that the filter capacitance $C_{f}$ is known. In addition, let the compensation control terms $\left(u_{\text {fad }}\right.$, $\left.u_{f a q}\right)$ and the feedback control terms $\left(u_{s m d}, u_{s m q}\right)$ be obtained by the following control laws as:

$$
\begin{array}{cl}
u_{f a d}=u_{d}, & u_{f a q}=u_{q} \\
u_{s m d}=-\tau_{d} \sigma_{d}-\varepsilon_{d} \operatorname{sgn}\left(\sigma_{d}\right), & u_{s m q}=-\tau_{q} \sigma_{q}-\varepsilon_{q} \operatorname{sgn}\left(\sigma_{q}\right) \\
\sigma_{d}=\left(e_{L d}+\gamma_{d} e_{i d}\right), & \sigma_{q}=\left(e_{L q}+\gamma_{q} e_{i q}\right)
\end{array}
$$

where $\sigma_{d}$ and $\sigma_{q}$ are sliding surfaces, and $\tau_{d}, \tau_{q}, \varepsilon_{d}$, and $\varepsilon_{q}$ are positive constants. Then $e_{L d}$ and $e_{L q}$ converge to zero.

Proof: The given control law (7) is known as the sliding-mode control term. Its stability analysis can be divided into two tasks. The first task is to determine the stability of the reduced-order sliding-mode dynamics, while the second task is to verify the reachability condition. By setting $\dot{\sigma}_{d}=\sigma_{d}=\dot{\sigma}_{q}=\sigma_{q}=0$, the second-order sliding-mode dynamics restricted to the sliding surface can be derived as:

$$
e_{L d}+C_{f} \gamma_{d} \dot{e}_{L d}=0, \quad e_{L q}+C_{f} \gamma_{q} \dot{e}_{L q}=0
$$

which is asymptotically stable. Therefore, it should be shown that the reachability condition is satisfied. To this end, the Lyapunov function $V_{0}(t)$ can be defined as:

$$
V_{0}(t)=\sigma_{d}^{2}+\sigma_{q}^{2}
$$

Its time derivative is expressed as:

$$
\dot{V}_{0}=2\left(\sigma_{d} \dot{\sigma}_{d}+\sigma_{q} \dot{\sigma}_{q}\right)
$$

The following equation can be obtained from (3), (6), (7), and (8):

$$
\dot{\sigma}_{d}=\frac{\gamma_{d}}{L_{f}}\left(v_{i n d}-u_{d}\right), \quad \dot{\sigma}_{q}=\frac{\gamma_{q}}{L_{f}}\left(v_{i n q}-u_{q}\right)
$$

Substituting (11) into (10) yields:

$$
\dot{V}_{0}=-2\left(\tau_{d} \frac{\gamma_{d}}{L_{f}} \sigma_{d}^{2}+\tau_{q} \frac{\gamma_{q}}{L_{f}} \sigma_{q}^{2}\right) \leq 0
$$

The above compensation control law (6) requires accurate knowledge of $u_{d}$ and $u_{q}$ due to the parameter uncertainties. Therefore, the following $i^{\text {th }}$ fuzzy rules for the two fuzzy models ( $\eta_{d}$ and $\eta_{q}$ ) are applied to approximate $u_{d}$ and $u_{q}$, respectively.

Fuzzy rule $i$ for $\eta_{d}$ : IF $x_{j}$ is $F_{j i}$, THEN $\eta_{d}$ is $S_{d i}$.

Fuzzy rule $i$ for $\eta_{q}$ : IF $x_{j}$ is $F_{j i}$, THEN $\eta_{q}$ is $S_{q i}$.

where $j=1,2,3,4$, and $i=1,2, \ldots, r$, and $r$ is the total number of fuzzy rules. $x_{j}$ represents the state variables (i.e., $x_{l}=v_{L d}$, $\left.x_{2}=v_{L q}, x_{3}=i_{i d}, x_{4}=i_{i q}\right) . F_{j i}$ denotes the fuzzy sets associated with $x_{j} . S_{d i}$ and $S_{q i}$ are the fuzzy singletons for $\eta_{d}$ and $\eta_{q}$, respectively. The membership functions $g_{j i}\left(x_{j}\right)$ are used to further characterize the fuzzy sets $F_{j i}$. The final output $\left(\eta_{d}, \eta_{q}\right)$ of the above fuzzy models can be inferred by using a standard fuzzy inference method that consists of a singleton fuzzifier, a product fuzzy inference, and a weighted average defuzzifier.

$$
\eta_{d}=\xi_{d}{ }^{T} h=\sum_{i=1}^{r} \xi_{d i} h_{i}, \quad \eta_{q}=\xi_{q}{ }^{T} h=\sum_{i=1}^{r} \xi_{q i} h_{i}
$$

where $\xi_{d}=\left[\xi_{d 1}, \cdots, \xi_{d r}\right]^{T}=\left[S_{d 1}, \cdots, S_{d r}\right]^{T}$ and $\xi_{q}=\left[\xi_{q 1}, \cdots, \xi_{q r}\right]^{T}$ $=\left[S_{q 1}, \cdots, S_{q r}\right]^{T}$ are the adaptable parameter vectors, and $h=$ $\left[h_{1}, \cdots, h_{r}\right]^{T}$ is the fuzzy basis function. Moreover, the vector $h_{i}$ is considered as the normalized weight of each IF-THEN rule, which satisfies both $h_{i} \geq 0$ and $\sum_{i=1}^{r} h_{i}=1$. Thus, $h_{i}$ can be expressed as:

$$
h_{i}=\frac{\prod_{j=1}^{4} g_{j i}\left(x_{j}\right)}{\sum_{k=1}^{r} \prod_{j=1}^{4} g_{j k}\left(x_{j}\right)}, \quad i=1,2, \ldots, r .
$$

It is assumed that $\xi_{*_{d}}$ and $\xi_{*_{q}}$ are the optimal parameter vectors. According to standard results [23], [24], a fuzzy system can uniformly approximate nonlinear functions to an arbitrary accuracy. As a result, if the searching spaces for $\eta_{d}$ and $\eta_{q}$ are sufficiently large, the following inequalities can be assumed:

$$
\begin{aligned}
& \varepsilon_{d} \geq\left|u_{d}-\eta_{d}\right|=\left|u_{d}-\xi_{*_{d}}^{T} h\right| \\
& \varepsilon_{q} \geq\left|u_{q}-\eta_{q}\right|=\left|u_{q}-\xi_{*_{q}}^{T} h\right|
\end{aligned}
$$

Now, the fuzzy adaptive compensation control terms ( $u_{\text {fad }}$, $u_{f a q)}$ and the sliding-mode feedback control terms $\left(u_{s m d}, u_{s m q}\right)$ can be written by: 


$$
\begin{gathered}
u_{f a d}=\xi_{d}{ }^{T} h=\sum_{i=1}^{r} \xi_{d i} h_{i}, \quad u_{f a q}=\xi_{q}{ }^{T} h=\sum_{i=1}^{r} \xi_{q i} h_{i} \\
\xi_{d i}=-\frac{1}{\lambda_{d i}} \int_{0}^{t} \sigma_{d} h_{i} d t, \quad \xi_{q i}=-\frac{1}{\lambda_{q i}} \int_{0}^{t} \sigma_{q} h_{i} d t \\
u_{s m d}=-\tau_{d} \sigma_{d}-\varepsilon_{d} \operatorname{sgn}\left(\sigma_{d}\right), \quad u_{s m q}=-\tau_{q} \sigma_{q}-\varepsilon_{q} \operatorname{sgn}\left(\sigma_{q}\right)
\end{gathered}
$$

where $\lambda_{d i}$ and $\lambda_{q i}$ are positive design parameters. Then, the following can be established:

Theorem 1: Assume that the filter capacitance $C_{f}$ is known. Let the control law be given by (16) and (17). Then $e_{i d}, e_{i q}, e_{L d}$, and $e_{L q}$ converge to zero, and $\xi_{d i}$ and $\xi_{q i}$ are bounded.

Proof: Since Proposition 1 indicates that the linear sliding surface (8) guarantees the asymptotic stability of the sliding-mode dynamics, it should be demonstrated that $\sigma_{d}$ and $\sigma_{q}$ converge to zero. The Lyapunov function can be defined as:

$$
V(t)=\sigma_{d}^{2}+\sigma_{q}^{2}+\sum_{i=1}^{r} \frac{\gamma_{d}}{L_{f}} \lambda_{d i} \widetilde{\xi}_{d i}^{2}+\sum_{i=1}^{r} \frac{\gamma_{q}}{L_{f}} \lambda_{q i} \widetilde{\xi}_{q i}^{2}
$$

where $\tilde{\xi}_{d i}=\xi_{* d i}-\xi_{d i}, \quad \tilde{\xi}_{q i}=\xi_{* q i}-\xi_{q i}$. Now the time derivative of (18) can be expressed as follows:

$$
\dot{V}=2\left(\sigma_{d} \dot{\sigma}_{d}+\sigma_{q} \dot{\sigma}_{q}-\sum_{i=1}^{r} \frac{\gamma_{d}}{L_{f}} \lambda_{d i} \widetilde{\xi}_{d i} \dot{\xi}_{d i}-\sum_{i=1}^{r} \frac{\gamma_{q}}{L_{f}} \lambda_{q i} \widetilde{\xi}_{q i} \dot{\xi}_{q i}\right)
$$

On the other hand, (16) and (17) imply that:

$$
\begin{gathered}
v_{\text {ind }}=-\tau_{d} \sigma_{d}-\varepsilon_{\mathrm{d}} \operatorname{sgn}\left(\sigma_{d}\right)+\xi_{d}{ }^{T} h \pm \xi_{* d}{ }^{T} h \\
v_{\text {inq }}=-\tau_{q} \sigma_{q}-\varepsilon_{\mathrm{q}} \operatorname{sgn}\left(\sigma_{q}\right)+\xi_{q}{ }^{T} h \pm \xi_{* q}{ }^{T} h \\
\dot{\xi}_{d i}=-\frac{1}{\lambda_{d i}} h_{i} \sigma_{d}, \quad \dot{\xi}_{q i}=-\frac{1}{\lambda_{q i}} h_{i} \sigma_{q} .
\end{gathered}
$$

Also, the following equation can be derived from (3) and (20):

$$
\begin{aligned}
& \dot{\sigma}_{d}=\frac{\gamma_{d}}{L_{f}}\left[-\tau_{d} \sigma_{d}-\varepsilon_{\mathrm{d}} \operatorname{sgn}\left(\sigma_{d}\right)+\widetilde{\xi}_{d}{ }^{T} h+\left(\xi_{* d}{ }^{T} h-u_{d}\right)\right] \\
& \dot{\sigma}_{q}=\frac{\gamma_{q}}{L_{f}}\left[-\tau_{q} \sigma_{q}-\varepsilon_{\mathrm{q}} \operatorname{sgn}\left(\sigma_{q}\right)+\tilde{\xi}_{q}{ }^{T} h+\left(\xi_{* q}{ }^{T} h-u_{q}\right)\right]
\end{aligned}
$$

If the inequalities in (15) are used, substituting (20), (21), and (22) into (19) yields:

$$
\begin{aligned}
\dot{V} \leq & -2 \frac{\gamma_{d}}{L_{f}}\left(\tau_{d} \sigma_{d}^{2}+\varepsilon_{\mathrm{d}}\left|\sigma_{d}\right|-\left(\xi_{*_{d}}^{T} h-u_{d}\right) \sigma_{d}\right) \\
& -2 \frac{\gamma_{q}}{L_{f}}\left(\tau_{q} \sigma_{q}^{2}+\varepsilon_{\mathrm{q}}\left|\sigma_{q}\right|-\left(\xi_{*_{q}}^{T} h-u_{q}\right) \sigma_{q}\right) . \\
& \leq-2\left(\tau_{d} \frac{\gamma_{d}}{L_{f}} \sigma_{d}^{2}+\tau_{q} \frac{\gamma_{q}}{L_{f}} \sigma_{q}^{2}\right) \leq 0
\end{aligned}
$$

By applying the integration on both sides of (23):

$$
\int_{0}^{\infty} \dot{V}(t) d t \leq-2\left(\tau_{d} \frac{\gamma_{d}}{L_{f}} \int_{0}^{\infty} \sigma_{d}^{2} d t+\tau_{q} \frac{\gamma_{q}}{L_{f}} \int_{0}^{\infty} \sigma_{q}^{2} d t\right)
$$

or:

$$
V(\infty)-V(0) \leq-2\left(\tau_{d} \frac{\gamma_{d}}{L_{f}} \int_{0}^{\infty} \sigma_{d}^{2} d t+\tau_{q} \frac{\gamma_{q}}{L_{f}} \int_{0}^{\infty} \sigma_{q}^{2} d t\right)
$$

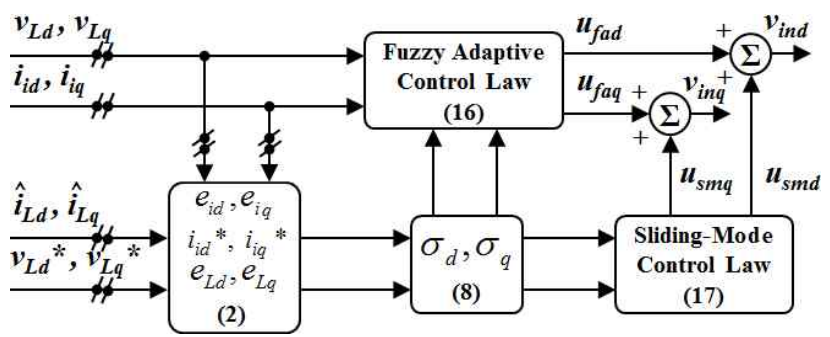

Fig. 2. Block diagram of the proposed FASVC.

Then, (25) can be redefined as:

$$
\begin{aligned}
2\left(\tau_{d} \frac{\gamma_{d}}{L_{f}} \int_{0}^{\infty} \sigma_{d}^{2} d t+\tau_{q} \frac{\gamma_{q}}{L_{f}} \int_{0}^{\infty} \sigma_{q}^{2} d t\right) & \leq V(0)-V(\infty) \\
& \leq V(0)
\end{aligned}
$$

where $V_{0}(t) \geq 0$ is used. Then, the following inequalities can be derived:

$$
\int_{0}^{\infty} \sigma_{d}^{2} d t<\infty, \quad \int_{0}^{\infty} \sigma_{q}^{2} d t<\infty
$$

which implies that $\sigma_{d}, \sigma_{q} \in L_{2}$. Since $\dot{V} \leq 0$ as shown in (23), $V(t)$ does not increase and is upper bounded as $V(t) \leq V(0)$. This entails that $\sigma_{d}, \sigma_{q} \in L_{\infty}$, and $\xi_{d i}, \xi_{q i} \in L_{\infty}$. Hence, it can be concluded by [21], [24], [25], and [26] that the closed-loop system is stable.

Fig. 2 shows a block diagram of the proposed FASVC. In this figure, the load current information is estimated through a conventional load current observer [27] (Kalman-Bucy optimal observer) to reduce the number of current sensors and enhance system reliability. Therefore, the load currents $\left(i_{L d}, i_{L q}\right)$ in (2) are substituted with their estimated values $\left(\hat{i}_{L d}, \hat{i}_{L q}\right)$, respectively.

Remark 1: This remark discusses the selection process of the controller gains. In this paper, for achieving a fast convergence and a rapid transient response, the adaptive gains are tuned to large values. According to (16), these adaptive gains are inversely proportional to $\lambda_{d i}$ and $\lambda_{q i}$. As a result, smaller values of $\lambda_{d i}$ and $\lambda_{q i}$ can result in larger values of the adaptive gains and vice versa. On the other hand, the sliding surfaces $\left(\sigma_{d}\right.$ and $\left.\sigma_{q}\right)$ are further defined to obtain the sliding-mode feedback control terms $\left(u_{s m d}\right.$ and $\left.u_{s m q}\right)$ given in (17), and these feedback control terms can be regarded as a PD controller. In this context, the control parameters $\gamma_{d}, \gamma_{q}, \tau_{d}$, and $\tau_{q}$ can be designed based on the tuning rule of [22]. As a final point, all of the control parameters $\left(\gamma_{d}, \gamma_{q}, \tau_{d}, \tau_{q}, \lambda_{d i}\right.$, and $\lambda_{q i}$ ) can be tuned according to the following steps: 1) Tune the parameters $\left(\gamma_{d}, \gamma_{q}, \tau_{d}\right.$, and $\left.\tau_{q}\right)$ based on the tuning rule of [22]; 2) Set quite large values for $\lambda_{d i}$ and $\left.\lambda_{q i} ; 3\right)$ Reduce $\lambda_{d i}$ and $\lambda_{q i}$ by a small amount; 4) End the tuning process if the present control parameters give satisfactory transient and steady-state performances, otherwise go back to Step 3 .

Remark 2: As described in (16) and (17), the proposed FASVC consists of two control terms: a fuzzy adaptive compensation control term $\left(u_{f a d}, u_{f a q}\right)$ and a sliding-mode feedback control term $\left(u_{s m d}, u_{s m q}\right)$. The first term takes parameter uncertainties into account. Meanwhile, the other 
TABLE I

PARAMETERS OF A Three-Phase UPS INVERTER

\begin{tabular}{|c|c|}
\hline Parameters & Values \\
\hline Rated power & $1 \mathrm{kVA}$ \\
Fundamental frequency $f$ & $60 \mathrm{~Hz}$ \\
Load output voltages $\boldsymbol{v}_{\text {Labc, rms }}$ & $110 \mathrm{~V}$ \\
Switching \& sampling frequency & $5 \mathrm{kHz}$ \\
Output filter capacitance $C_{f}$ & $6.5 \mu \mathrm{F}$ \\
Output filter inductance $L_{f}$ & $10 \mathrm{mH}$ \\
DC-link voltage $V_{D C}$ & $295 \mathrm{~V}$ \\
\hline
\end{tabular}

term stabilizes the error dynamics of the system. Therefore, the proposed control scheme can achieve good performance in the existence of the parameter uncertainties.

\section{EXPERIMENTAL VALIDATION}

\section{A. Prototype Overview}

To verify the performance of the proposed control scheme, a prototype $1 \mathrm{kVA}$ three-phase UPS inverter system with a TMS320F28335 DSP is tested. The parameters of the three-phase UPS inverter are listed in Table I. Note that the values of the $L C$ output filter have a cutoff frequency of 620 Hz. Normally, larger values of the filter elements $\left(L_{f}\right.$ and $\left.C_{f}\right)$ can realize better filter performance. On the other hand, large values of $L_{f}$ and $C_{f}$ can increase the system cost and volume. In addition, a large current flows into $C_{f}$ even under no load. Therefore, selecting the $L C$ output filter parameters always follows a trade-off between their pros and cons. The guidelines for choosing an $L C$ output filter for the pulse width modulation inverters are available in [28].

A complete block diagram of a three-phase UPS inverter system with the proposed FASVC is shown in Fig. 3. In this figure, both the inverter currents $\left(\boldsymbol{i}_{i a b c}\right)$ and load voltages $\left(v_{L a b c}\right)$ in the stationary $a b c$ reference frame are sensed, and then transformed to values in the synchronously rotating $d q$ reference frame by Park's transformation $\left(\boldsymbol{v}_{L d q}, \boldsymbol{i}_{i d q}\right)$. These values are first used at the conventional load current observer. After that, the estimated load currents $\left(\hat{i}_{L d}, \hat{i}_{L q}\right)$ and the reference load voltages $\left(v_{L d}{ }^{*}, v_{L q}{ }^{*}\right)$ are injected into the proposed FASVC. Then, after the $d q$-axis voltage control inputs $\left(v_{\text {ind }}, v_{\text {inq }}\right)$ are converted to quantities $\left(v_{i n \alpha}, v_{\text {in } \beta}\right)$ in the stationary $\alpha \beta$ reference frame using an inverse Park's transformation. Six gate pulses are generated to drive the three-phase UPS inverter by using the space vector pulse-width modulation (SVPWM) technique with sampling and switching frequencies of $5 \mathrm{kHz}$.

\section{B. Fuzzy Rules and Controller Gains}

In order to create the fuzzy models $\eta_{d}$ and $\eta_{q}$ given in (16), the total numbers of fuzzy rules for the four state variables $\left(x_{l}\right.$, $x_{2}, x_{3}$, and $x_{4}$ ) are optimized as:

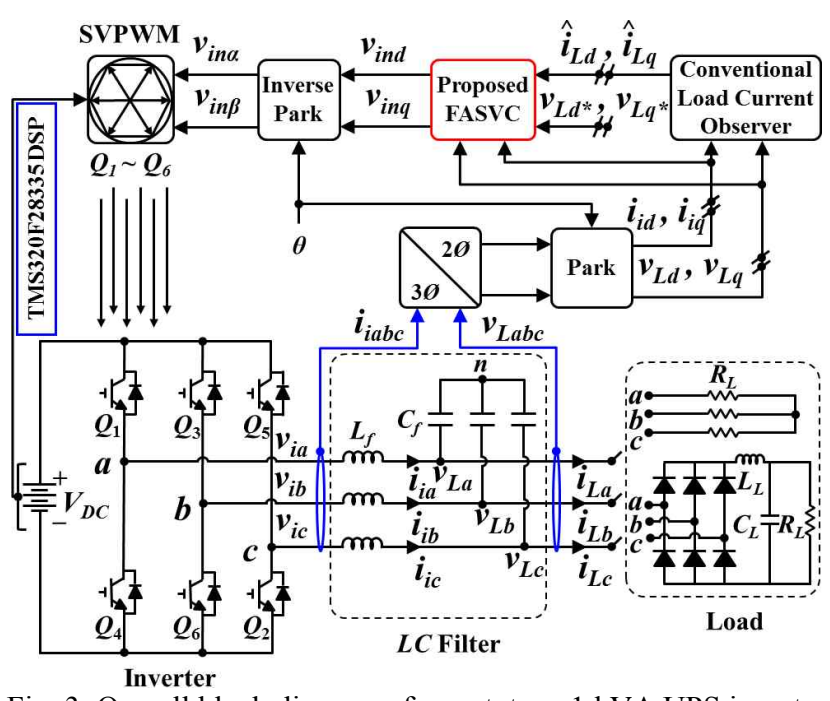

Fig. 3. Overall block diagram of a prototype $1 \mathrm{kVA}$ UPS inverter system.

$$
r=(F)^{m}=(2)^{4}=16
$$

where $F$ denotes the fuzzy sets and $m$ is the number of state varaibles.

The fuzzy sets are characterized by two linguistic terms (negative $N$ and positive $P$ ) for each state variable and designed in the form of the following membership functions:

$$
\begin{gathered}
g_{N 1}\left(x_{1}\right)=e^{-\left(x_{1}+160\right)^{2} /(320)^{2}}, g_{P 1}\left(x_{1}\right)=e^{-\left(x_{1}-160\right)^{2} /(320)^{2}} \\
g_{N 2}\left(x_{2}\right)=e^{-\left(x_{2}+5\right)^{2} /(10)^{2}}, g_{P 2}\left(x_{2}\right)=e^{-\left(x_{2}-5\right)^{2} /(10)^{2}} \\
g_{N 3}\left(x_{3}\right)=e^{-\left(x_{3}+6\right)^{2} /(12)^{2}}, g_{P 3}\left(x_{3}\right)=e^{-\left(x_{3}-6\right)^{2} /(12)^{2}} \\
g_{N 4}\left(x_{4}\right)=e^{-\left(x_{4}+2\right)^{2} /(4)^{2}}, g_{P 4}\left(x_{4}\right)=e^{\left.-\left(x_{4}-2\right)^{2} / 4\right)^{2}}
\end{gathered}
$$

where the Gaussian functions are utilized as membership functions due to their simplicity. In addition, the constant values $(160,5,6$, and 2$)$ are chosen by considering the constraints of each state variable with a small additional tolerance.

Briefly, the sixteen fuzzy rules for $\eta_{d}$ are:

$r_{l}$ : IF $x_{1}$ is $N_{l}$ and $x_{2}$ is $N_{2}$ and $x_{3}$ is $N_{3}$ and $x_{4}$ is $N_{4}$, THEN $\eta_{d}$ is $\xi_{d l}$. $r_{2}$ : IF $x_{1}$ is $N_{l}$ and $x_{2}$ is $N_{2}$ and $x_{3}$ is $N_{3}$ and $x_{4}$ is $P_{4}$, THEN $\eta_{d}$ is $\xi_{d 2}$. $r_{3}$ : IF $x_{1}$ is $N_{l}$ and $x_{2}$ is $N_{2}$ and $x_{3}$ is $P_{3}$ and $x_{4}$ is $N_{4}$, THEN $\eta_{d}$ is $\xi_{d 3}$.

$$
\text { • }
$$

$r_{16}$ : IF $x_{1}$ is $P_{1}$ and $x_{2}$ is $P_{2}$ and $\dot{x_{3}}$ is $P_{3}$ and $x_{4}$ is $P_{4}$, THEN $\eta_{d}$ is $\xi_{d 16}$.

In addition, the sixteen fuzzy rules for $\eta_{q}$ are: $r_{l}$ : IF $x_{1}$ is $N_{l}$ and $x_{2}$ is $N_{2}$ and $x_{3}$ is $N_{3}$ and $x_{4}$ is $N_{4}$, THEN $\eta_{q}$ is $\xi_{q l}$. $r_{2}$ : IF $x_{1}$ is $N_{l}$ and $x_{2}$ is $N_{2}$ and $x_{3}$ is $N_{3}$ and $x_{4}$ is $P_{4}$, THEN $\eta_{q}$ is $\xi_{q 2}$. $r_{3}$ : IF $x_{1}$ is $N_{1}$ and $x_{2}$ is $N_{2}$ and $x_{3}$ is $P_{3}$ and $x_{4} i$ is $N_{4}$, THEN $\eta_{q}$ is $\xi_{q 3}$.

$$
r_{16} \text { IF } x_{1} \text { is } P_{1} \text { and } x_{2} \text { is } P_{2} \text { and } \dot{x_{3}} \text { is } P_{3} \text { and } x_{4} \text { is } P_{4} \text {, THEN } \eta_{q} \text { is }
$$


Finally, the controller gains are tuned via extensive simulation studies based on Remark 1 as: $\gamma_{d}=\gamma_{q}=130, \tau_{d}=\tau_{q}$ $=15, \varepsilon_{d}=\varepsilon_{q}=70$, and $\lambda_{d i}=\lambda_{q i}=55 \times 10^{-5}$.

\section{Comparative Experimental Results}

Experimental results are demonstrated under two scenarios to fully highlight the transient and steady-state performances of the proposed FASVC as compared to the conventional SMC. Scenario 1 shows the transient-state response and the steady-state response when a three-phase resistive load with a $40 \Omega$ resistance is instantaneously applied, i.e., no load to full load. Scenario 2 reveals the steady-state response when a three-phase diode rectifier is applied to the load terminals, which has the following $R L C$ values: $R_{L}=90 \Omega, L_{L}=10 \mathrm{mH}$, and $C_{L}=60 \mu \mathrm{F}$. Meanwhile, Scenario 3 presents the performance at the steady-state when an unbalanced resistive load is connected to the inverter output terminals, i.e., only phase $c$ is opened. In this paper, $30 \%$ reductions in both $L_{f}$ and $C_{f}$ are assumed as the parameter uncertainties under each scenario to clearly demonstrate the transient and steady-state performances of the proposed FASVC and the conventional SMC.

Figs. 4 and 5 demonstrate the relative experimental results of both the proposed and the conventional control strategies under the three scenarios mentioned above. Both figures illustrate the waveforms of the load phase voltages ( $v_{L a}, v_{L b}$, $\left.v_{L c}\right)$, inverter phase currents $\left(i_{i a}, i_{i b}, i_{i c}\right)$, and estimated load phase currents $\left(\hat{i}_{L a}, \hat{i}_{L b}, \hat{i}_{L c}\right)$. Note that only $\left(i_{i a}\right.$ and $\left.\hat{i}_{L a}\right)$ for Scenarios $1 \& 2$ and $\left(i_{i a}, i_{i b}, i_{i c}\right.$ and $\left.\hat{i}_{L a}, \hat{i}_{L b}, \hat{i}_{L c}\right)$ for Scenario 3 are exposed. Based on Figs. 4 and 5, the experimental results can be described as follows:

Figs. 4(a) and 5(a) show the dynamic responses for Scenario 1. Fig. 4(a) depicts that the load voltage waveforms are slightly distorted and recovered to the steady-state within $0.5 \mathrm{~ms}$. Meanwhile, Fig. 5(a) shows that it takes $1.2 \mathrm{~ms}$ for the load voltage waveforms to be restored to the steady-state. In Figs. 4(b) and 5(b), the steady-state performances under Scenario 2 are depicted. More specifically, it can be seen that the proposed FASVC has pure sinusoidal load voltage waveforms with a smaller THD (1.08\%) than the conventional SMC (2.83\%). Figs. 4(c) and 5(c) elaborate the responses under Scenario 3 at the steady-state. The load voltage waveforms presented in Fig. 4(c) attain a $0.47 \%$ lower THD value and reflect a sinusoidal behavior with $1.32 \%$ less steady-state error when compared with the voltage waveforms shown in Fig. 5(c).

Table II summarizes the THDs and steady-state rms errors of both the proposed FASVC and the conventional SMC after each scenario reaches the steady-state condition. It can be seen in Table II that under all three scenarios, the THDs and steady-state errors of the proposed FASVC (i.e., about $1.10 \%$ and $0.50 \%$, respectively) are significantly improved when

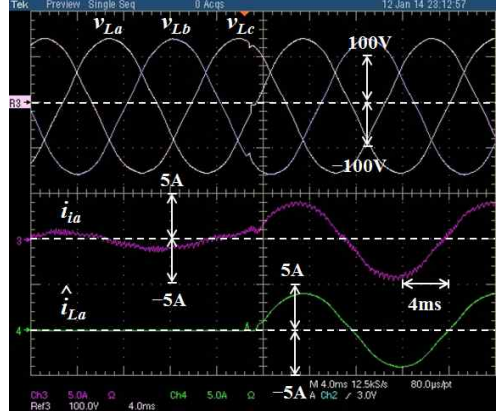

(a)

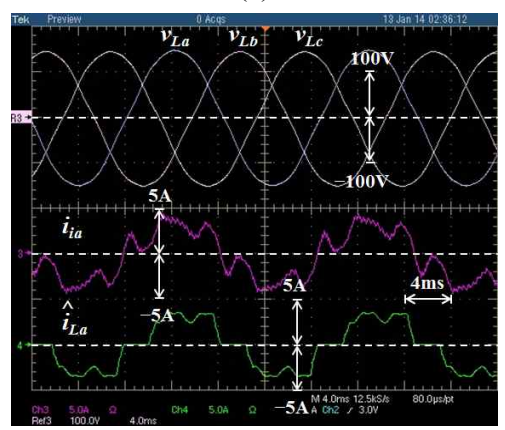

(b)

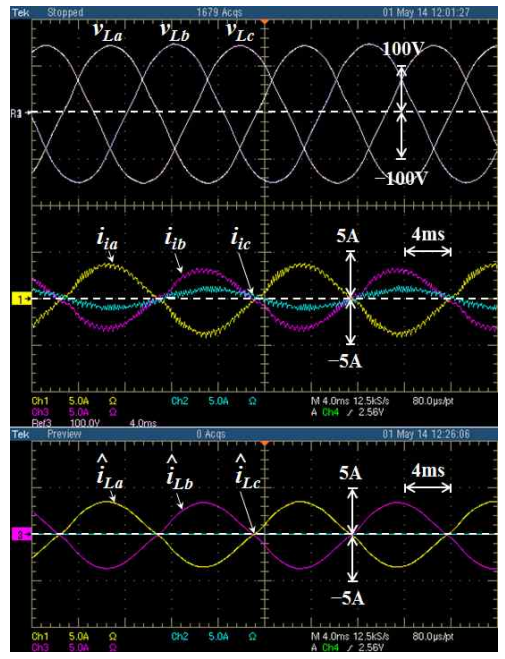

(c)

Fig. 4. Experimental results of the proposed FASVC with $-30 \%$ parameter uncertainties in $L_{f}$ and $C_{f}$. (a) Sudden load disturbance (i.e., $0 \%$ to $100 \%$ ). (b) Nonlinear load (i.e., Crest factor $=1.62$ ). (c) Unbalanced load (i.e., Phase $c$ open).

TABLE II

STEADY-STATE PERFormanCES OF COMPARATIVE EXPERIMENTAL RESULTS

\begin{tabular}{|c|c|c|c|c|}
\hline \multirow{2}{*}{ Scenarios } & \multicolumn{2}{|c|}{ THD (\%) } & \multicolumn{2}{c|}{ Steady-state error (\%) } \\
\cline { 2 - 5 } & $\begin{array}{c}\text { Proposed } \\
\text { FASVC }\end{array}$ & $\begin{array}{c}\text { Conventional } \\
\text { SMC }\end{array}$ & $\begin{array}{c}\text { Proposed } \\
\text { FASVC }\end{array}$ & $\begin{array}{c}\text { Conventional } \\
\text { SMC }\end{array}$ \\
\hline 1 & 0.35 & 0.75 & 0.09 & 1.30 \\
\hline 2 & 1.08 & 2.83 & 0.45 & 2.27 \\
\hline 3 & 0.40 & 0.87 & 0.13 & 1.45 \\
\hline
\end{tabular}




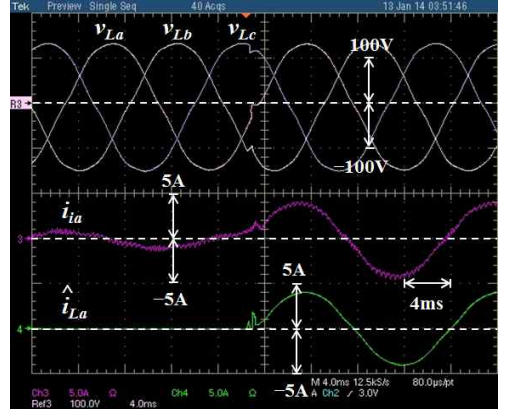

(a)

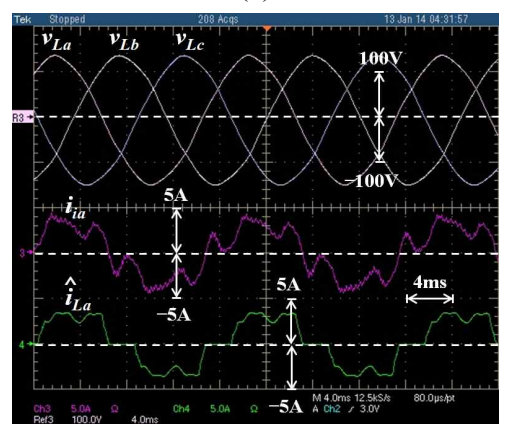

(b)

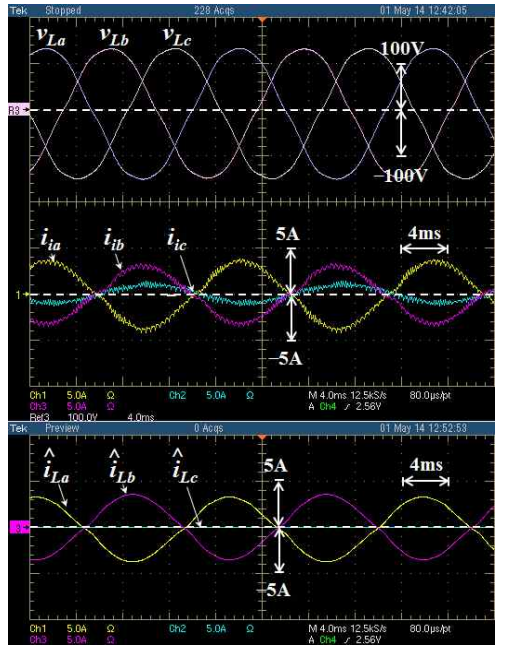

(c)

Fig. 5. Experimental results of the conventional SMC with $-30 \%$ parameter uncertainties in $L_{f}$ and $C_{f}$. (a) Sudden load disturbance (i.e., $0 \%$ to $100 \%$ ). (b) Nonlinear load (i.e., Crest factor $=1.62$ ). (c) Unbalanced load (i.e., Phase $c$ open).

compared to the conventional SMC (i.e., about $2.90 \%$ and $2.30 \%$, respectively).

\section{CONCLUSIONS}

In this paper, a combined fuzzy adaptive sliding-mode voltage controller (FASVC) was proposed for a three-phase UPS inverter. The proposed FASVC was insensitive to parameter uncertainties due to the use of a fuzzy adaptive compensation control term. In addition, the error dynamics of the system were stabilized by a sliding-mode feedback control term. The stability of the proposed method was analytically proven by the Lyapunov theory. To evaluate the performance of the proposed FASVC, a prototype $1 \mathrm{kVA}$ three-phase UPS inverter test-bed with a TMS320F28335 DSP was constructed and tested. Then, the outstanding performances (i.e., quicker voltage recovery time after a step load change, reduced THD under a nonlinear load, and smaller steady-state error for an unbalanced load) of the proposed control technique were verified through a comparison with the results obtained from a conventional SMC under three different load scenarios, and in the presence of parameter uncertainties.

\section{ACKNOWLEDGMENT}

This work was supported by the National Research Foundation of Korea (NRF) grant funded by the Korea government (MSIP) (No. 2012R1A2A2A01045312).

\section{REFERENCES}

[1] S. Roy and L. Umanand, "Integrated magnetics-based multisource quality ac power supply," IEEE Trans. Ind. Electron., Vol. 58, No. 4, pp. 1350-1358, Apr. 2011.

[2] J. H. Han, K. Lee, C. S. Song, G. Jang, G. Byeon, and C. H. Park, "A new assessment for the total harmonic contributions at the point of common coupling," J. Electr. Eng. Technol., Vol. 9, No. 1, pp. 6-14, Jan. 2014.

[3] H. K. Kang, C. H. Yoo, I. Y. Chung, D. J. Won, and S. I. Moon, "Intelligent coordination method of multiple distributed resources for harmonic current compensation in a microgrid," J. Electr. Eng. Technol., Vol. 7, No. 6, pp. 834-844, Nov. 2012.

[4] J. H. Lee, H. G. Jeong, and K. B. Lee, "Performance improvement of grid-connected inverter systems under unbalanced and distorted grid voltage by using a PR controller," J. Electr. Eng. Technol., Vol. 7, No. 6, pp. 918-925, Nov. 2012.

[5] C. Salim, B. M. Toufik, and G. Amar, "Harmonic current compensation based on three-phase three-level shunt active filter using fuzzy logic current controller," J. Electr. Eng. Technol., Vol. 6, No. 5, pp. 595-604, Sep. 2011.

[6] B. Zhao, Q. Song, W. Liu, and Y. Xiao, "Next-generation multi-functional modular intelligent UPS system for smart grid," IEEE Trans. Ind. Electron., Vol. 60, No. 9, pp. 3602-3618, Sep. 2013.

[7] D. E. Kim and D. C. Lee, "Feedback linearization control of three-phase UPS inverter systems," IEEE Trans. Ind. Electron., Vol. 57, No. 3, pp. 963-968, Mar. 2010.

[8] G. Escobar, A. M. Stankovic, and P. Mattavelli, "An adaptive controller in stationary reference frame for D-statcom in unbalanced operation," IEEE Trans. Ind. Electron., Vol. 51, No. 2, pp. 401-409, Apr. 2004.

[9] R. Escobar, A. A. Valdez, J. Leyva-Ramos, and P. Mattavelli, "Repetitive-based controller for a UPS inverter to compensate unbalance and harmonic distortion," IEEE Trans. Ind. Electron., Vol. 54, No. 1, pp. 504-510, Feb. 2007.

[10] P. Cortés, G. Ortiz, J. I. Yuz, J. Rodrisguez, S. Vazquez, and L. G. Franquelo, "Model predictive control of an 
inverter with output $L C$ filter for UPS applications," IEEE Trans. Ind. Electron., Vol. 56, No. 6, pp. 1875-1883, Jun. 2009.

[11] C. Li, S. M. Ji, and D. P. Tan, "Multiple-loop digital control method for a $400-\mathrm{Hz}$ inverter system based on phase feedback," IEEE Trans. Power Electron., Vol. 28, No. 1, pp. 408-417, Jan. 2013.

[12] P. Mattaveli, "An improved deadbeat control for UPS using disturbance observers," IEEE Trans. Ind. Electron., Vol. 52, No. 1, pp. 206-212, Feb. 2005.

[13] T. S. Lee, S. J. Chiang, and J. M. Chang, " $H_{\infty}$ loop-shaping controller designs for the single-phase UPS inverters," IEEE Trans. Power Electron., Vol. 16, No. 4, pp. 473-481, Jul. 2001.

[14] F. Y. Hsu and L. C. Fu, "Adaptive fuzzy control for uninterruptible power supply with three-phase PWM inverter," in Proc. IEEE Fuzzy Systems Symposium, pp. 188-193, 1996.

[15] D. S. Xu, K. Yong, and C. Jian, "An algorithm for the output waveform compensation of SPWM inverters based on fuzzy-repetitive control," J. Electr. Eng., Vol. 55, No. 3-4, pp. 64-70, 2004.

[16] O. Kukrer, H. Komurcugil, and A. Doganalp, "A three-level hysteresis function approach to the sliding-mode control of single-phase UPS inverters," IEEE Trans. Ind. Electron., Vol. 56, No. 9, pp. 3477-3486, Sep. 2009.

[17] H. Komurcugil, "Rotating sliding line based sliding-mode control for single-phase UPS inverters," IEEE Trans. Ind. Electron., Vol. 59, No. 10, pp. 3719-3726, Oct. 2012.

[18] R. J. Wai and C. Y. Lin, "Dual active low-frequency ripple control for clean-energy power-conditioning mechanism," IEEE Trans. Ind. Electron., Vol. 58, No. 11, pp. 5172-5185, Nov. 2011

[19] M. Dai, M. N. Marwali, J. W. Jung, and A. Keyhani, "A three-phase four-wire inverter control technique for a single distributed generation unit in island mode," IEEE Trans. Power Electron., Vol. 23, No. 1, pp. 322-331, Jan. 2008.

[20] M. N. Marwali and A. Keyhani, "Control of distributed generation systems-part I: voltages and currents control," IEEE Trans. Power Electron., Vol. 19, No. 6, pp. 1541-1550, Nov. 2004

[21] F. L. Lewis, C. T. Abdallah, and D. M. Dawson, Control of Robot Manipulators, Macmillan, Ch. 2, 1993.

[22] K. J. Astrom and B. Witternmark, Computer-Controlled Systems: Theory and Design, Prentice Hall, Ch. 8, 1990.

[23] J. L. Castro, "Fuzzy logic controllers are universal approximators," IEEE Trans. Syst. Man. Cybern., Vol. 25, No. 4, pp. 629-635, Apr.1995.

[24] L. X. Wang, "Stable adaptive fuzzy control of nonlinear systems," IEEE Trans. Fuzzy Syst., Vol. 1, No. 2, pp. 146-155, May 1993.

[25] H. H. Choi, "Adaptive controller design for uncertain fuzzy systems using variable structure control approach," Automatica, Vol. 45, No. 11, pp. 2646-2650, Nov. 2009.

[26] W. S. Lin and C. S. Chen, "Robust adaptive sliding-mode control using fuzzy modelling for a class of uncertain MIMO nonlinear systems," IEE Proc. Electr. Power Appl., Vol. 149, No. 149, pp. 193-201, May 2002.

[27] F. Lin, Robust Control Design: An Optimal Control Approach, John Willey \& Sons, Ch. 4, 2007.

[28] H. Kim and S. K. Sul, "A novel filter design for output $L C$ filters of PWM inverters," Journal of Power Electronics, Vol. 11, No. 1, pp. 74-81, Jan. 2011.

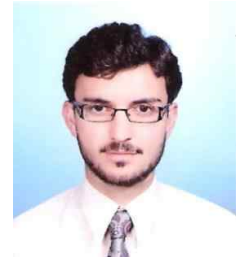

Khawar Naheem received his B.S. degree in Electrical Power Engineering from the Comsats Institute of Information Technology (CIIT), Abbottabad, Pakistan, in 2011. He is currently pursuing his M.S. degree in the Division of Electronics and Electrical Engineering, Dongguk University, Seoul, Korea. His current research interests are the control of distributed generation systems using renewable energy sources, electric vehicles (EVs), micro/smart-grid systems, and DSP-based electric machine drives.

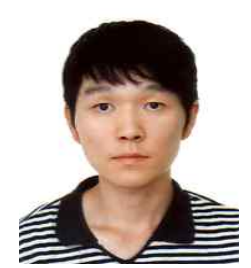

Young-Sik Choi received his B.S. degree in Electrical Engineering from Dongguk University, Seoul, Korea in 2009. He is presently pursuing his $\mathrm{Ph} . \mathrm{D}$. degree in the Division of Electronics and Electrical Engineering, Dongguk University. His current research interests include power conversion systems and drives for electric

vehicles (EVs).

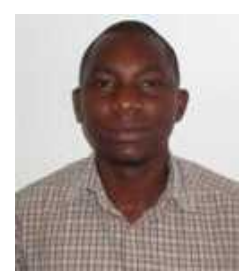

Francis Mwasilu received his B.S. degree in Electrical Engineering from the University of Dar es Salaam, Dar es Salaam, Tanzania, in 2008. From 2009 to 2011, he worked as a Utilities Engineer at JTI-Tanzania. He is presently pursuing his $\mathrm{Ph} . \mathrm{D}$. degree in the Division of Electronics and Electrical Engineering, Dongguk University, Seoul, Korea. His current research interests include distributed generation systems, electric vehicles (EVs), and renewable energy sources integration in modern power systems.

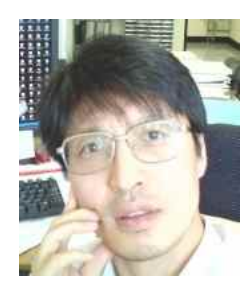

Han Ho Choi received his B.S. degree in Control and Instrumentation Engineering from Seoul National University (SNU), Seoul, Korea, in 1988, and his M.S. and $\mathrm{Ph}$.D. degrees in Electrical Engineering from the Korea Advanced Institute of Science and Technology (KAIST), Daejeon, Korea, in 1990 and 1994, respectively. He is currently with the Division of Electronics and Electrical Engineering, Dongguk University, Seoul, Korea. His current research interests include control theory and its application to real world problems.

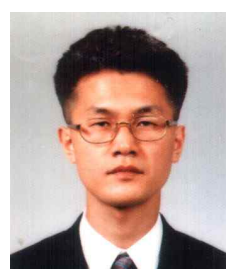

Jin-Woo Jung received his B.S. and M.S. degrees in Electrical Engineering from Hanyang University, Seoul, Korea, in 1991 and 1997, respectively. He received his Ph.D. degree in Electrical and Computer Engineering from The Ohio State University, Columbus, Ohio, USA, in 2005. From 1997 to 2000, he was with the Home Appliance Research Laboratory, LG Electronics Co., Ltd., Seoul, Korea. From 2005 to 2008, he worked at the R\&D Center and with the PDP Development Team, Samsung SDI Co., Ltd., Korea, as a Senior Engineer. Since 2008, he has been an Associate Professor with the Division of Electronics and Electrical Engineering, Dongguk University, Seoul, Korea. His current research interests include DSP-based electric machine drives, distributed generation systems using renewable energy sources, and power conversion systems and drives for electric vehicles (EVs). 